\title{
A FIXED-POINT THEOREM
}

\section{A. D. WALlaCE}

Introduction. The purpose of this note is to sharpen a recent result of G. E. Schweigert [4]. ${ }^{1}$ It will be shown that the condition of semi local-connectedness may be dropped. However, if this is strengthened to local-connectedness, then the conclusion asserts the existence of a fixed point. Further, though perhaps of less interest, it is shown that separability is not necessary.

In a second section we give a somewhat more abstract version which is valid for certain partially ordered topological spaces. So far as is known this is the first result of this type to appear in the literature.

1. Schweigert's theorem. It is assumed that $S$ is a compact (that is, bicompact) Hausdorff space, connected and nondegenerate. Moreover $T$ is a topological transformation of $S$ onto itself, $T S=S$.

THEOREM. If $e$ is an end point of $S$ fixed under $T$, then there exists a continuum $K \subset S-e$ invariant under $T$. Further, no point of $S$ separates any pair of points of $K$ in $S$.

Proof. Since $e$ is an end point it is readily seen that we can find a point $y$ such that

(1) $S=A+B, \quad e \in A, \quad y+T^{-1} y \subset B, \quad A \cdot B=z \in S$,

with $A$ and $B$ nondegenerate proper subcontinua of $S$. We infer that

(2) $S=T A+T B, \quad e \in T A, \quad y \in T B, \quad T A \cdot T B=T z$,

so that $S=(A+T A)+B \cdot T B$. Clearly $A+T A$ is a continuum and hence $[5]$ so is

$$
(A+T A) \cdot B \cdot T B=z \cdot T B+T z \cdot B .
$$

We then have (supposing that $z \neq T z$ ) either (a) $z \in T B$ and $T z \in S-B$ or (b) $T z \in B$ and $z \in S-T B$. In the first case $T^{-1} z \in B$ and we are able to apply the same argument that we use in the second case if we use $T^{-1}$ in place of $T$. We therefore assume (b) and readily verify that $A \subset T A, T B \subset B$.

Using induction it follows that we may write

Received by the editors December 29, 1944.

${ }^{1}$ Numbers in brackets refer to the Bibliography at the end of the paper. 


$$
\begin{gathered}
S=T^{n} A+T^{n} B, \quad e \in T^{n} A, \quad T^{n+1} z \in T^{n} B, \quad T^{n} z=T^{n} A \cdot T^{n} B, \\
T^{n-1} A \subset T^{n} A, \quad T^{n} B \subset T^{n-1} B .
\end{gathered}
$$

If $X$ is the union of the sets $T^{n} A$ and $Y$ is the intersection of the sets $T^{n} B$ it may be shown that $X$ is connected and $K=\bar{X} \cdot Y$ is a continuum [5]. Clearly $T \bar{X}=\bar{T} \bar{X}, T Y=Y$ so that $K$ is an invariant continuum contained in $S-e$. Suppose that $p$ and $q$ are points of $K$ separated by $r$ in $S$ :

$$
S-r=U+V, \quad p \in U, \quad q \in V, \quad U \mid V .
$$

We may admit that $e$ is in $U$. For some $m$ and all $n \geqq m$ it is clear that both $U$ and $V$ intersect $T^{n} A$. Thus, this set being connected, we infer that $r$ is a point of $T^{n} A$ and hence that $Y$ meets $T^{n} A$ and consequently $Y$ has a point in common with $X$. But this would imply that for some $n, T^{n+1} z=T^{n} z$, giving $T z=z$.

Corollary. There exists a continuum $H \subset S-e$, having no cut point and invariant under $T$.

This follows at once if we observe that an argument advanced by Kelley [2] to improve a theorem of Ayres [1] is unnecessarily restricted by the assumption of separability. The basic material necessary to modify Kelley's proof will be found in $\$ \S 1$ and 3 of [5]. In particular the generalized Brouwer theorem is given on p. 488. See also the references to Milgram, Moore, and Tukey and, further, Kuratowski [3].

Corollary. If $S$ is locally connected there exists a fixed point distinct from $e$.

PROOF. With the notation employed in the proof of the theorem suppose that $p$ and $q$ are distinct points and let $P$ and $Q$ be disjoint open connected sets containing $p$ and $q$ and lying in $S-e$. For some $m$ and all $n \geqq m$ both $P$ and $Q$ intersect $T^{n} A$. Thus $P$ meets both $T^{n} A$ and $T^{n} B$ and so contains $T^{n} z$. Similarly $Q$ contains $T^{n} z$. This is a contradiction.

It is a simple matter to show that Schweigert's theorem follows from the one given above. We need only use the same argument he gives to pass from the invariant node to the case of the fixed end point.

2. A generalization. In this section it is assumed that $P$ is a compact Hausdorff space containing more than one point and, in addition, that we are given a binary relation $\Delta$ on $P$. We suppose that $\Delta$ is reflexive and transitive so that we have (a) $x \Delta x$ for each $x$ in $P$ and 
(b) $x \Delta y$ and $y \Delta z$ imply $x \Delta z$. On the topological side let $M(a)[N(a)]$ denote the set of all $x$ such that $x \Delta a[a \Delta x]$ holds. We then assume that $\Delta$ is semi-continuous (that is, both lower and upper semi-continuous) in the sense that

(i) For each $a$ in $P$ the sets $M(a)$ and $N(a)$ are closed.

A non-empty set $Q$ is termed $\Delta$-simple if for any pair $q_{1}, q_{2}$ of $Q$ we have either $q_{1} \Delta q_{2}$ or $q_{2} \Delta q_{1}$. In virtue of a result due to Hausdorff (or directly by transfinite induction) it is readily seen that there exists a maximal $\Delta$-simple set containing any given $\Delta$-simple set. An element $p$ is called a maximal element for a set $R$ if $r \in R$ and $p \Delta r$ imply $r \Delta p$.

Every maximal $\Delta$-simple set is closed.

For if $Q$ is such a set then $Q(q)=M(q)+N(q)$ is closed for each $q \in Q$ and it is not difficult to see that $Q$ is the intersection of all the sets $Q(q)$ for all $q \in Q$.

Each non-void closed subset of $P$ contains a maximal element.

It is legitimate to suppose that the set concerned is $P$ itself, so let $Q$ be a maximal $\Delta$-simple set. For $q_{1}, q_{2}$ in $Q$ one set of the pair $N\left(q_{1}\right)$, $N\left(q_{2}\right)$ is a subset of the other. For each $q$ the set $N(q)$ is closed and in virtue of the compactness of $P$ there is a point $p$ common to all these sets. The point $p$ is in $Q$. If $x$ is a point of $Q$ we have $x \Delta p$ since $p$ is in the set $N(x)$. Suppose that for $x$ in $P-Q$ we have $p \Delta x$. Since $Q$ is maximal $\Delta$-simple there is a point $y$ in $Q$ such that both $x \Delta y$ and $y \Delta x$ fail to hold. But by transitivity $p \Delta x$ and $y \Delta p$ imply $y \Delta x$, a contradiction.

Three further assumptions must be made about $P$ :

(ii) There exists a unique element $e$ in $P$ such that we have $e \Delta x$ for each $x$.

(iii) Each set $M(a)$ is $\Delta$-simple.

(iv) For any $x, y$ distinct from $e$ there is a $z$ such that $z \Delta x, z \Delta y$ hold but $z \neq e$.

It follows at once that

If $Q$ is maximal $\Delta$-simple then $a \in Q$ implies $M(a) \subset Q$.

For if $q \in Q$ and $x \in M(a)$ then $a \Delta q$ and transitivity imply $x \Delta q$. If $q \Delta a$ holds then $q \in M(a)$ and by (iii) we have either $x \Delta q$ or $q \Delta x$ so that $x$ is an element of $Q$ by maximality.

From (iv) we see that

Any pair of maximal $\Delta$-simple sets $X, Y$ have in common an element $z \neq e$.

To prove this let $x, y$ be elements of $X-e, Y-e$ respectively. Let $z$ be given by (iv). Then $z$ is in $M(x)$ and so in $X$. Similarly $z$ is in $Y$.

Theorem. If $T$ is a homeomorphism of $P$ onto itself and both $T$ and 
$T^{-1}$ preserve the relation $\Delta$, then there exists an $f \neq e$ such that both $T f \Delta f$ and $f \Delta T f$ hold.

Proof. Let $C$ be a maximal $\Delta$-simple set. Then $T C$ is a set of the same type. Let $z$ be an element common to $C$ and $T C$ and distinct from $e$. Both $z$ and $T z$ are in $T C$ so that we must have either $z \Delta T z$ or $T z \Delta z$. Now the argument we employ for the former case can be used in the latter provided we replace $T$ by $T^{-1}$ so that we assume that we have $z \Delta T z$. Let $Z$ be the set $z, T z, T^{2} z, \ldots$. It is clear that if $D$ is any maximal $\Delta$-simple set containing $Z$, then $T D$ has these same properties. Let $X$ be the intersection of all the maximal $\Delta$-simple sets containing $Z$. Then $X$ is closed, $\Delta$-simple, and further satisfies the condition $T X=X$. Let $f$ be a maximal element of $X$. Both $T f$ and $T^{-1} f$ are in $X$ and so we have $T f \Delta f$ and $T^{-1} f \Delta f$. The latter implies $f \Delta T f$. This completes the proof.

COROLLARY. If $\Delta$ is symmetric then there is a fixed point distinct from $e_{2}$

The second corollary to the theorem of $\$ 1$ follows if we define $\Delta$ as follows: $x \Delta y$ holds if $x=e, x=y$ or $x$ separates $y$ from $e$ in $S$.

\section{BIBLIOGRAPHY}

1. W. L. Ayres, Some generalizations of the Scherrer fixed point theorems, Fund. Math. vol. 16 (1930) p. 333.

2. J. L. Kelley, Fixed sets under homeomorphisms, Duke Math. J. vol. 5 (1939) pp. 535 and 536.

3. C. Kuratowski, Une methode d'elimination des nombres transfinis des raisonnements mathematigues, Fund. Math. vol. 3 (1922) pp. 76-108.

4. G. E. Schweigert, Fixed elements and periodic types for homeomorphisms on S. L. C. continua, Amer. J. Math. vol. 66 (1944) p. 231.

5. A. D. Wallace, Monotone transformations, Duke Math. J. vol. 9 (1942) pp. 487506.

The University of Pennsyluania 